\title{
Design Guidelines for PVT Collectors
}

1 Transparent cover - Optiona

Blocks IR radiation, protects from wind, snow, hail, acid rain High transparency, UV resistance, anti-reflective, mechanical stability, durability, protective

Tempered glass, polymer

Not present for WISC design / when operated at low temperature, e.g. as source for heat pump system

Front cover of PV module

Protects cells, barrier against moisture

High transparency, UV resistance, long lasting, temperature stable, low dilatation coefficient, low IR emission

Tempered glass, glass, polyme

b Encapsulant

protects cells, maintains cells

Good thermal conductivity, long lasting, resilient to

dilatation and shear effect, easy lamination if not sprayed,

low temperature coefficient

EVA, polyolefins, silicone, polymer

\section{Solar PV cells connected}

C electricity production, absorbing IR, transmitting heat

High efficiency, high absorptance, low IR emission, low heat

resistance, low temperature coefficient, soldering and tabbing

adapted, thin , bifacial if absorber design allows

$\mathrm{c}-\mathrm{Si}$, a-Si, CIGS, Organic

4 Encapsulant - Option

rotects cells, maintains cells

Good thermal conductivity, long lasting, resilient

to dilatation and shear effect, easy lamination,

low temperature coefficient

EVA, polyolefins, silicone, polymer

5a Backsheet / Rear cover of PV module - Optiona

Prects cells, maintain cells, barrier against moisture, glue PV and

Good thermal conductivity, long lasting, resilient to dilatation and shear

effect, low temperature coefficient

Tempered glass, polymer

Not necessary in a fully glued PVT but part of PV industrial modules

5b Glue/Encapsulant - Optiona

Glues PV and T

Good thermal conductivity, long lasting, resilient to dilatation and shear

effect, low temperature coefficient

Not nocessary in a PVT mechanically fixed where the backsheet is necessary

Exploded-view of a typical PVT collector
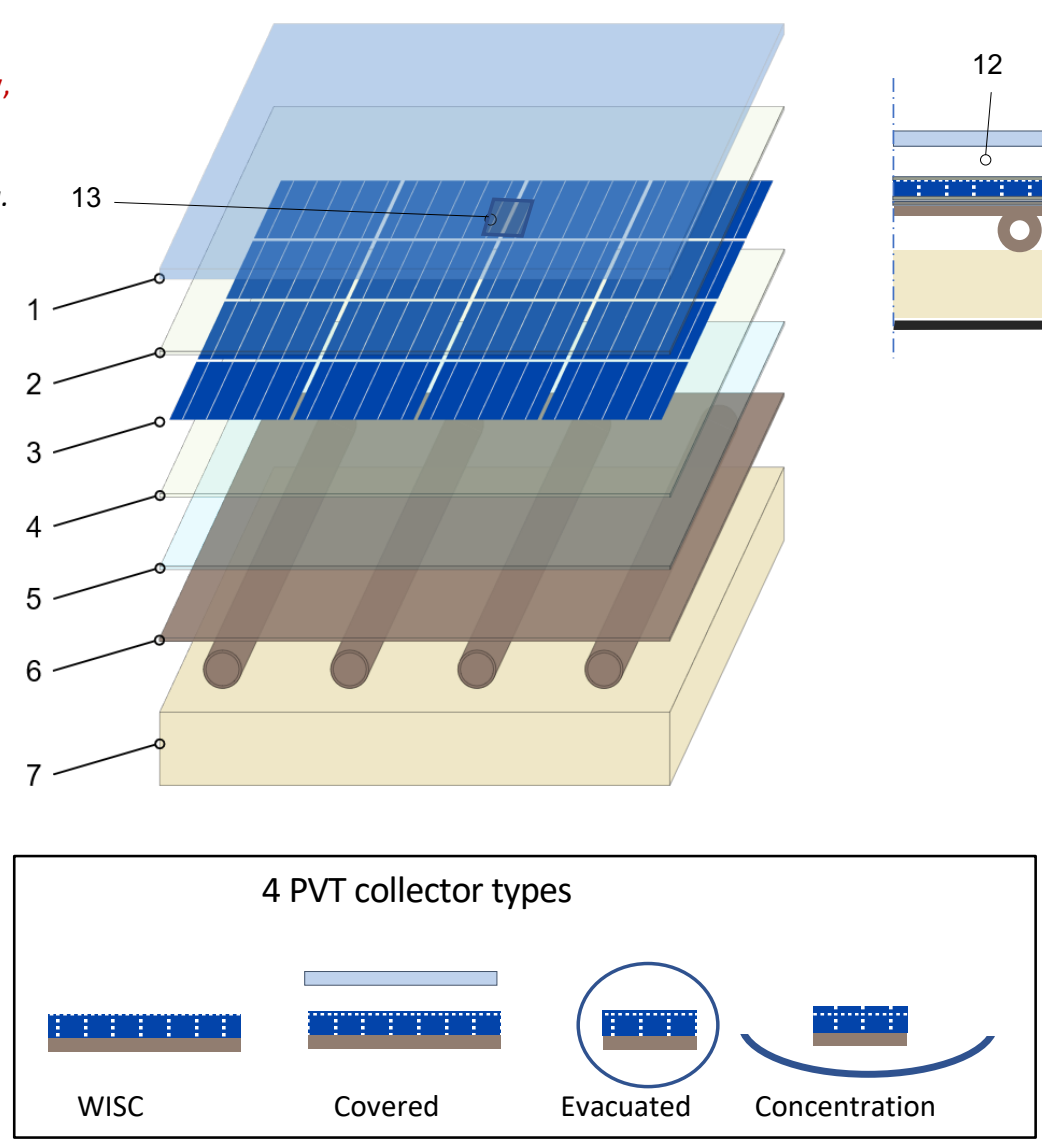

Cross section of a covered flat plate PVT collecto

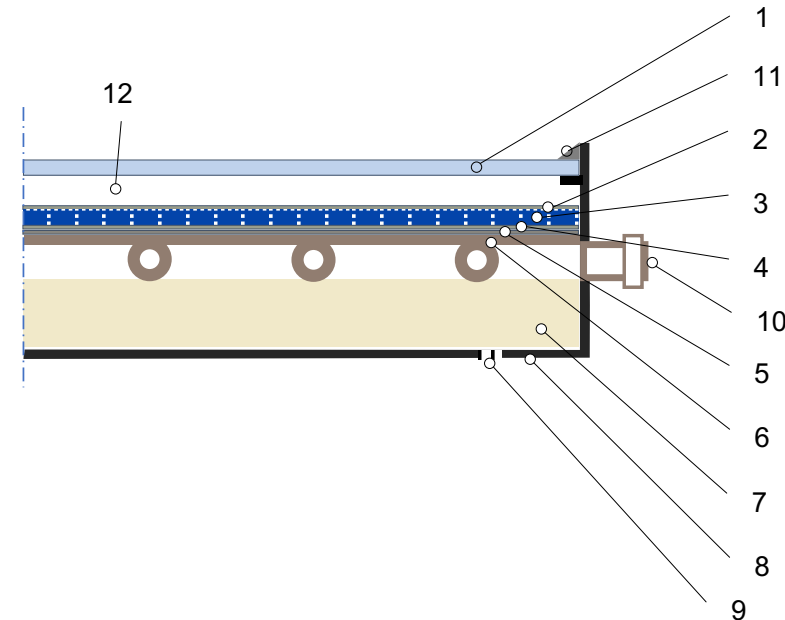

Examples of 3 WISC designs No cover
No insulation

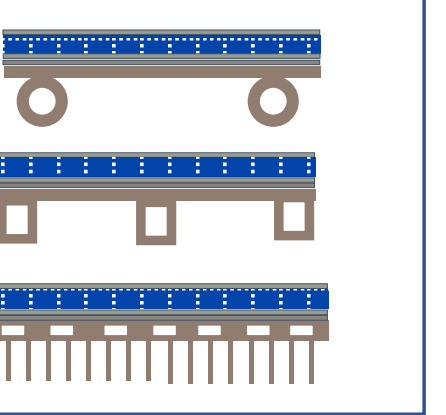

6a Absorber

Absorbs solar radiation and heat from the PV, transfers heat to the heat transfer medium Low IR emission, good contact with upper layer, high heat transfer with ambient for WISC PVT, light-weight, thin, easy to weld or moulded or extruded,

high thermal conductivity, thin for lamination, low pressure drop, low inertia, low temperature coefficient, high heat transfer to fluid properties, eventually transparent Copper, Aluminium, Steel, Polymer

Very high exchange surface with ambient for non-insulated WISC PVT when operated with a heat pump

6b. Heat transfer medium

Extracts thermal energy, cools down collector

Open loop or closed loop, single or multipass, opaque or not

Liquid

Gaseous Bi fluids

Water

Glycol/Water Carbon Dioxide

Additive Other special

Hydrocarbon gasses
Refrigerant

Ranofluids
7 Insulation - Optional

Reduces heat losses, stays hydrophobic

Low thermal conductivity, hydrophobic, insects resistant,

no degassing, not flammable

Mineral wool or similar

Optional: with a protective foil on top

8 Casing - Optional

Protects, rigidifies, simplifies fixing, maintains air tightness,

supports all elements

Light, stiff, watertight, formable, corrosion free, non sensitive

to electrical currents, lets components dilatation occur, gas

filling possible

Aluminium, polymer

9 Air vent - Optional

Ventilates at low pace, dehumidifies

Insects protected, accessible to visual inspection,

adapted flow rate of air through

10 Fluid Outlets

Connect the absorber with circuit

Easy to clip or connect to next collector, no spiky parts, consider forces on tubing if handled hereby, considers corrosion risks if made in another metal than absorber Metallic piping materials (Cu, stainless steel, Al, ...), polymer, clips

11 Sealing

Water tightness

UV resistant, highly resilient, easy to put in place and to

dismount

Silicone, elastomers

12 Gap - Optional (only if 1 is present)

Reduces top heat losses by convection, conduction, protects cells

Air, inert gas, transparent insulation materials

13 Junction box

Connects strings of cells to DC cables outside the module, standard PV connectors

Temperature resistant (incl. sealing), corrosion resistant,

absorber material compatible

Polymer and glue 